\title{
Increased migraine risk in osteoporosis patients: a nationwide population-based study
}

\author{
Chieh-Hsin Wu ${ }^{3,4}$, Zi-Hao Zhang ${ }^{5 \dagger}$, Ming-Kung Wu ${ }^{6 \dagger}$, Chiu-Huan Wang ${ }^{7}$, Ying-Yi Lu ${ }^{1,2^{*}}$ and Chih-Lung Lin ${ }^{4,8^{*}}$
}

\begin{abstract}
Background: Osteoporosis and migraine are both important public health problems and may have overlapping pathophysiological mechanisms. The aim of this study was to use a Taiwanese population-based dataset to assess migraine risk in osteoporosis patients.

Methods: The Taiwan National Health Insurance Research Database was used to analyse data for 40,672 patients aged $\geq 20$ years who had been diagnosed with osteoporosis during 1996-2010. An additional 40,672 age-matched patients without osteoporosis were randomly selected as the non-osteoporosis group. The relationship between osteoporosis and migraine risk was estimated using Cox proportional hazard regression models.
\end{abstract}

Results: During the follow-up period, 1110 patients with osteoporosis and 750 patients without osteoporosis developed migraine. After controlling for covariates, the overall incidence of migraine was 1.37-fold higher in the osteoporosis group than in the non-osteoporosis group (3.72 vs. 1.24 per 1000 person-years, respectively). Migraine risk factors included high Charlson Comorbidity Index score, female gender, hypertension, depression, asthma, allergic rhinitis, obesity, and tobacco use disorder.

Conclusions: Our results indicate that patients with a history of osteoporosis had a higher risk of migraine.

Keywords: Osteoporosis, Migraine, Nationwide population-based study

\section{Background}

Both osteoporosis and migraine are common conditions that can affect quality of life and can impose large social and economic burdens (Kuo et al. 2015; Manandhar et al. 2015a, b; Mbewe et al. 2015; Rao et al. 2015; Steiner et al. 2015; Lampl et al. 2016). The National Institutes of Health Consensus Development Conference Statement defines osteoporosis as a skeletal disorder characterized by diminished bone strength resulting in increased fracture risk. Bone strength is measured in terms of both bone mineral density (BMD) and bone quality (Nih Consensus Development Panel on Osteoporosis Prevention and Therapy 2001). In elderly populations, osteoporosis

\footnotetext{
*Correspondence: actinp@hotmail.com; chihlung1@yahoo.com

${ }^{\dagger}$ Zi-Hao Zhang and Ming-Kung Wu have contributed equally to this work ${ }^{1}$ Department of Dermatology, Kaohsiung Veterans General Hospital, No. 386 Dazhong 1st Rd, Kaohsiung 81362, Taiwan

${ }^{4}$ Department of Neurosurgery, Kaohsiung Medical University Hospital, Kaohsiung Medical University, No. 100 Tzyou 1st Road, Kaohsiung 80708, Taiwan

Full list of author information is available at the end of the article
}

affects approximately $30 \%$ of women and $12 \%$ of men (Rachner et al. 2011). Migraine is a neurological disorder that manifests as a debilitating headache associated with altered sensory perception (Goadsby et al. 2002; Charles 2013; Baykan et al. 2015; Manandhar et al. 2015a, b). The International Headache Society defines migraine as a headache that lasts for $4-72 \mathrm{~h}$ and has at least two of the following characteristics: pulsating quality, unilateral localization, moderate-to-severe pain intensity, and aggravation by movement (Headache Classification Subcommittee of the International Headache 2004). Previous studies have identified interacting relationships among migraine, various sleep disorders, depression, psoriasis, restless legs syndrome and cardiovascular disease (Kelman and Rains 2005; Pompili et al. 2009; Schurks et al. 2009; Cho et al. 2015; Egeberg et al. 2015; Kim et al. 2016; Risal et al. 2016). Migraine is associated with episodes of local sterile meningealinflammation, hypersensitized pain pathways, and increased inflammatory cytokines that contribute to the pathogenesis of osteoporosis, such 
as interleukins (ILs) or tumor necrosis factor- $\alpha$ (Braun and Schett 2012; Egeberg et al. 2015). Like osteoporosis and other inflammatory conditions, migraine is also apparently associated with systemic endothelial dysfunction (Vanmolkot et al. 2007; Sacco et al. 2013; Steyers and Miller 2014). Although no recent studies have suggested a link between osteoporosis and migraine, both conditions are independent risk factors for cardiovascular disease (Sumino et al. 2008; Bigal et al. 2010; Hyder et al. 2010), and both are comorbid with pain-related and psychiatric conditions (Kalaydjian and Merikangas 2008; Radaei et al. 2014). We therefore investigated the impact of osteoporosis on migraine risk in a nationwide cohort in Taiwan.

\section{Methods}

Database

This population-based cohort study used data obtained from the Taiwan National Health Insurance Research Database (NHIRD) maintained by the national health care system of Taiwan. The NHIRD is an encrypted secondary database containing medical data for approximately $99 \%$ of the 23.74 million residents of Taiwan. The Taiwan national health insurance program allows researchers to access this database of administrative data for patients. The NHIRD files are composed of comprehensive use and enrollment information of the patients. Besides, under regulations of the Personal Electronic Data Protection Law of Taiwan, all citizens and hospital identities in the NHIRD database were decoded. This retrospective cohort study analysed 1996-2010 data contained in a subset of the NHIRD, the Longitudinal Health Insurance Database 2010, which comprises data for 1 million beneficiaries randomly sampled from the primary NHIRD. In this study, diseases were identified and classified according to the diagnostic codes of the International Classification of Diseases, Ninth Revision, Clinical Modification (ICD-9-CM).

\section{Ethical approval}

The study was performed in accordance with the Declaration of Helsinki guidelines and was also evaluated and approved by the Institutional Review Board of Kaohsiung Medical University Hospital (KMUHIRB-EXEMPT (II)-20160016).

\section{Study population}

The study cohort included 40,672 patients aged 20 years or older who had been diagnosed with osteoporosis (ICD-9-CM code 733.0) during 1996-2010. To maximize accuracy, the analysis was limited to patients who had a record of $\geq 2$ osteoporosis diagnoses during ambulatory visits or $\geq 1$ diagnoses during inpatient care, an ICD-9 code assigned by an orthopaedist, and at least one BMD examination. The index date was defined as the date of the first clinical visit for osteoporosis. A migraine (ICD9-CM code 346) was defined as a history of two or more migraine diagnoses in ambulatory visits or one or more migraine diagnoses in inpatient care as well as a record of an ICD-9 code for migraine assigned by a neurologist. The date of diagnosis was defined as the date of the first diagnosis of osteoporosis. Matching control subjects were assigned an identical "pseudo date of diagnosis", which was the date of diagnosis in matched cases. The exclusion criteria were diagnosis of migraine before or on the index date, incomplete information, and age younger than 20 years.

The ratio of osteoporosis to non-osteoporosis patients was kept at 1:1 to enhance the power of statistical tests and to obtain a sufficient number of migraine cases for stratified analyses. The patients in the non-osteoporosis cohort were selected by a simple random sampling method in which one insured patient without osteoporosis was randomly selected and frequency matched with each person in the osteoporosis cohort in the same period by age, gender, and year of osteoporosis diagnosis. A post hoc sample size was calculated to determine statistical power. Logistic regression analysis was used to obtain a 4-digit match of the propensity score for each patient with the covariates, including age and gender. As a result, 40,672 subjects were enrolled in the non-osteoporosis cohort. Figure 1 shows a flowchart of the study procedure.

\section{Outcome and comorbidities}

The patients in both the osteoporosis and non-osteoporosis cohorts were followed up until diagnosis with

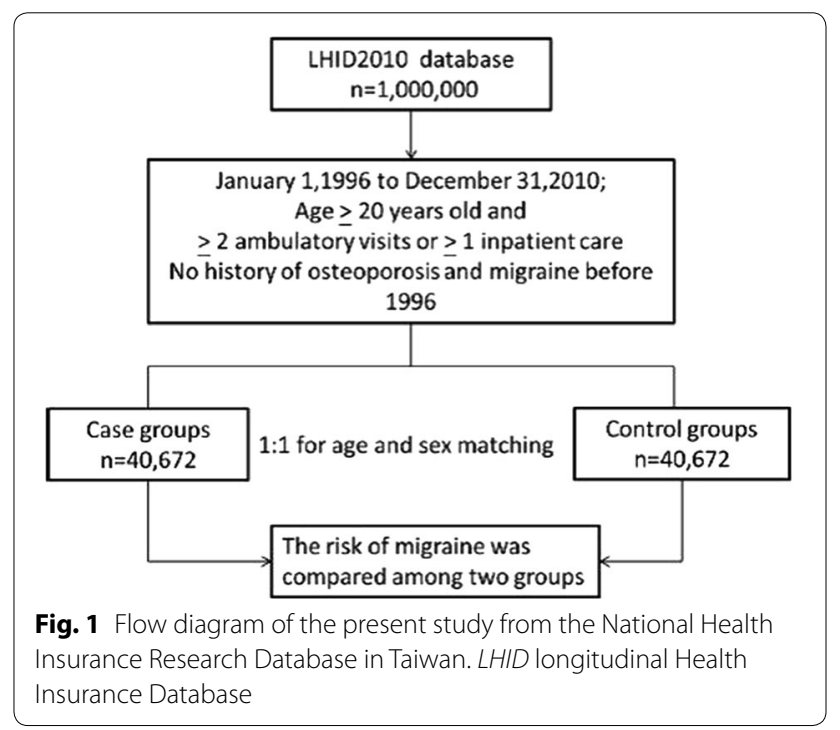


migraine, withdrawal from insurance, or the end of 2010, whichever occurred first. Baseline comorbidities identified by ICD-9-CM codes in the claims records before the index date included hypertension (ICD-9-CM codes 401-405), diabetes mellitus (ICD-9-CM code 250), hyperlipidemia (ICD-9-CM code 272), depression (ICD-9-CM codes 296.2, 296.3, 300.4 and 311), asthma (ICD-9-CM code 493), allergic rhinitis (ICD-9-CM code 477), psoriasis (ICD-9-CM code 696.1), obesity (ICD-9-CM code 278), tobacco use disorder (ICD-9-CM code 350.1), and alcohol attributed disease (ICD-9-CM codes 291.0-9, 303, 305.0, 357.5, 425.5, 535.3, 571.0-3, 980.0 and V11.3). The Charlson Comorbidity Index (CCI) score was used to assess the severity of comorbidities, i.e., myocardial infarction, congestive heart failure, peripheral vascular disease, cerebrovascular disease, dementia, chronic pulmonary disease, rheumatic disease, peptic ulcer disease, liver disease (mild, moderate, or severe), diabetes (with or without chronic complication), hemiplegia or paraplegia, renal disease, any malignancy (including lymphoma and leukemia but excluding skin malignancy), metastatic solid tumor, human immunodeficiency virus infection and acquired immune deficiency syndrome. The CCI scores were then categorized into four levels: $0,1-2,3-4$ and $\geq 5$.

\section{Statistical analyses}

Chi square test was used to compare distributions of categorical demographics and clinical characteristics between the osteoporosis and non-osteoporosis cohorts. The Student $t$ test and Wilcoxon rank-sum test were used as appropriate to compare mean age and followup time (y) between the two cohorts. The Kaplan-Meier method was used to estimate cumulative incidence, and the differences between the curves were tested by 2 -tailed log-rank test. For osteoporosis patients, survival was calculated until hospitalization, an ambulatory visit for migraine, or the end of the study period (December $31,2010)$, whichever occurred first. Incidence rates of migraine estimated in 1000 person-years were compared between the two cohorts. Univariable and multivariable Cox proportional hazard regression models were used to calculate hazard ratios (HRs) and $95 \%$ confidence intervals (CIs) for migraine if the proportional hazards assumption was satisfied. The multivariable Cox models were adjusted for age, gender, CCI score, and relevant comorbidities. A 2 -tailed $P$ value of $<0.05$ was considered statistically significant. All data processing and statistical analyses were performed using Statistical Analysis Software, version 9.4 (SAS Institute, Cary, NC, USA).

\section{Results}

Baseline characteristics of patients with and without osteoporosis

The baseline demographic characteristics and comorbidities in the two cohorts are presented in Table 1. In the osteoporosis cohort, $82.48 \%$ patients were female. Compared to the non-osteoporosis cohort, the osteoporosis cohort had significantly higher percentages of patients with hypertension (70.12 vs. $54.07, \mathrm{P}<0.001)$, diabetes mellitus (38.58 vs. $27.71, \mathrm{P}<0.001)$, hyperlipidemia (61.28 vs. $43.96, \mathrm{P}<0.001)$, depression (21.21 vs. 11.71 , $\mathrm{P}<0.001)$, asthma (28.77 vs. $19.20, \mathrm{P}<0.001)$, allergic rhinitis (44.62 vs. $32.38, \mathrm{P}<0.001)$, psoriasis $(2.15$ vs. 1.43 , $\mathrm{P}<0.001$ ), obesity ( 2.64 vs. $2.11, \mathrm{P}<0.001)$, tobacco use disorder (1.91 vs. $1.04, \mathrm{P}<0.001)$ and alcohol-attributable disease (2.63 vs. $1.92, \mathrm{P}<0.001)$. The osteoporosis cohort also had higher CCI scores. During a median observation time of 3.5 years, $2.73 \%$ (1110) of the osteoporosis patients had migraine (interquartile range [IQR] 1.5-6.2). The migraine incidence of osteoporosis cohort was significantly $(\mathrm{P}<0.001)$ higher than that in the non-osteoporosis patients (751 with migraine out of 40,672 age- and gender-matched controls [1.85 \%]) during a median observation time of 7.2 years [IQR 4.8-10.5]). Migraine development was significantly faster in the osteoporosis group (3.5 years) compared to the non-osteoporosis group ( 7.2 years) for the respective observation periods.

\section{Migraine incidence and risk}

The migraine incidence and HRs by gender, age and comorbidity are stratified in Table 2. During the follow-up period, migraine developed in $2.73 \%$ (1110) of the osteoporosis patients and in $1.85 \%$ (750) of the non-osteoporosis patients. The overall migraine risk was 1.37 times greater in the osteoporosis group compared to the non-osteoporosis group (3.72 vs. 1.24 per 1000 person-years, respectively) after adjusting for age, gender, $\mathrm{CCI}$, and related comorbidities (hypertension, diabetes mellitus, hyperlipidemia, depression, asthma, allergic rhinitis, psoriasis, obesity, tobacco use disorder and alcohol-attributable disease).

The gender-specific analyses showed that, in both cohorts, the incidence of osteoporosis was higher in women than in men (3.61 vs. 2.28 per 1000 person-years, respectively, in the osteoporosis cohort; 1.35 vs. 0.73 per 1000 person-years, respectively, in the non-osteoporosis cohort). Additionally, the osteoporosis group had a significantly higher migraine risk in both genders (adjusted HR 1.34, 95 \% CI 1.19-1.49 for women; adjusted HR 1.66, 95 \% CI 1.19-2.31 for men).

Although the incidence of migraine was consistently higher in all age groups in the osteoporosis cohort 
Table 1 Baseline characteristics of patients with and without osteoporosis

\begin{tabular}{|c|c|c|c|}
\hline \multirow[t]{2}{*}{ Variables } & \multicolumn{2}{|l|}{ Osteoporosis } & \multirow[t]{2}{*}{$P$ value } \\
\hline & Yes $(\mathrm{N}=40,672)$ & No $(N=40,672)$ & \\
\hline Migraine patients, n (\%) & $1110(2.73)$ & $751(1.85)$ & $<0.001$ \\
\hline Period of developing migraine, median (IQR), years & $3.5(1.5-6.2)$ & $7.2(4.8-10.5)$ & $<0.001$ \\
\hline Mean age at diagnosis of migraine, years & $57.9(10.4)$ & $62.6(10.3)$ & $<0.001$ \\
\hline \multicolumn{4}{|l|}{ Age group, $\mathrm{n}(\%)$} \\
\hline $20-49$ & $7361(18.10)$ & $7361(18.10)$ & \\
\hline$\geq 50$ & $33,311(81.90)$ & $33,311(81.90)$ & 1.000 \\
\hline \multicolumn{4}{|l|}{ Gender, n (\%) } \\
\hline Men & $7125(17.52)$ & $7125(17.52)$ & \\
\hline Women & $33,547(82.48)$ & $33,547(82.48)$ & 1.000 \\
\hline \multicolumn{4}{|l|}{ Charlson Comorbidity Index, n (\%) } \\
\hline 0 & $1912(4.70)$ & 7744 (19.04) & \\
\hline $1-2$ & $9667(23.77)$ & $14,437(35.50)$ & \\
\hline $3-4$ & $11,911(29.29)$ & $9861(24.25)$ & \\
\hline$\geq 5$ & $17,182(42.25)$ & $8630(21.22)$ & $<0.001$ \\
\hline \multicolumn{4}{|l|}{ Co-morbidity, n (\%) } \\
\hline Hypertension & $28,521(70.12)$ & 21,991 (54.07) & $<0.001$ \\
\hline Diabetes mellitus & $15,693(38.58)$ & $11,272(27.71)$ & $<0.001$ \\
\hline Hyperlipidemia & $24,923(61.28)$ & $17,879(43.96)$ & $<0.001$ \\
\hline Depression & $8625(21.21)$ & $4764(11.71)$ & $<0.001$ \\
\hline Asthma & $11,700(28.77)$ & 7807 (19.20) & $<0.001$ \\
\hline Allergic rhinitis & $18,146(44.62)$ & $13,168(32.38)$ & $<0.001$ \\
\hline Psoriasis & $874(2.15)$ & $583(1.43)$ & $<0.001$ \\
\hline Obesity & $1073(2.64)$ & $857(2.11)$ & $<0.001$ \\
\hline Tobacco use disorder & $776(1.91)$ & $422(1.04)$ & $<0.001$ \\
\hline Alcohol attributed disease & $1069(2.63)$ & $780(1.92)$ & $<0.001$ \\
\hline
\end{tabular}

$I Q R$ interquartile range, SD standard deviation

compared to the non-osteoporosis cohort, the migraine risk decreased with age. Age-specific risk comparisons showed that, compared to the non-osteoporosis cohort, the osteoporosis cohort had a significantly higher migraine risk in patients under 50 years old (adjusted HR 1.46, $95 \%$ CI 1.17-1.82, P < 0.001) than in patients over 50 years old (HR 1.34, 95 \% CI 1.20$1.50, \mathrm{P}<0.001)$. Regardless of comorbidities, migraine risk was higher in osteoporosis patients than in nonosteoporosis patients. However, the migraine risk contributed by osteoporosis decreased in the presence of comorbidity.

The Kaplan-Meier curves for the cumulative incidence of migraine between the osteoporosis and non-osteoporosis groups at the 15-year follow up are compared in Fig. 2. The Kaplan-Meier curves showed a significantly higher cumulative incidence of migraine in the osteoporosis cohort compared to the non-osteoporosis cohort (log-rank test $\mathrm{P}<0.001$ ).

\section{Risks factors for migraine in osteoporosis patients}

The Cox regression analysis revealed the following risk factors for migraine in the osteoporosis group: high CCI score, female gender, hypertension, depression, asthma, allergic rhinitis, obesity, and tobacco use disorder. Risk factors for migraine were female gender (adjusted HR 1.51, 95 \% CI 1.23-1.85), hypertension (adjusted HR 1.19, $95 \%$ CI 1.02-1.38), depression (adjusted HR 2.36, $95 \%$ CI 2.09-2.66), asthma (adjusted HR 1.28, 95 \% CI 1.131.45), allergic rhinitis (adjusted HR 1.46, 95 \% CI 1.291.66), obesity (adjusted HR 1.79, 95 \% CI 1.40-2.28), tobacco use disorder (adjusted HR 2.30, 95 \% CI 1.782.97) and high CCI (adjusted HR 1.57, 95 \% CI 1.44-1.71) (Table 3).

\section{Discussion}

To our knowledge, this is the first nationwide populationbased study of the relationship between osteoporosis and subsequent migraine in an Asian population. During the 
Table 2 Incidence and hazard ratios of migraine by demographic characteristics and comorbidity among patients with or without osteoporosis

\begin{tabular}{|c|c|c|c|c|c|c|c|c|}
\hline \multirow[t]{2}{*}{ Variables } & \multicolumn{3}{|c|}{ Patients with osteoporosis } & \multicolumn{3}{|c|}{ Patients without osteoporosis } & \multicolumn{2}{|c|}{ Compared to non- osteoporosis } \\
\hline & Migraine & PYs & Rate & Migraine & PYs & Rate & CrudeHR $^{\mathrm{a}}(95 \% \mathrm{Cl})$ & $\begin{array}{l}\text { Adjusted HR }{ }^{\mathrm{a}}(95 \% \\
\text { Cl) }\end{array}$ \\
\hline Overall & 1110 & $324,126.20$ & 3.42 & 751 & $604,550.99$ & 1.24 & $2.48(2.25-2.73)^{c}$ & $1.37(1.23-1.51)^{c}$ \\
\hline \multicolumn{9}{|l|}{ Gender } \\
\hline Men & 104 & $45,604.93$ & 2.28 & 78 & $106,313.43$ & 0.73 & $2.80(2.04-3.85)^{c}$ & $1.66(1.19-2.31)^{d}$ \\
\hline Women & 1006 & $278,521.27$ & 3.61 & 573 & $498,237.56$ & 1.35 & $2.39(2.17-2.66)^{c}$ & $1.34(1.19-1.49)^{c}$ \\
\hline \multicolumn{9}{|c|}{ Stratify by age } \\
\hline $20-49$ & 252 & $64,474.34$ & 3.91 & 130 & $109,506.02$ & 1.19 & $2.97(2.39-3.68)^{c}$ & $1.46(1.17-1.82)^{c}$ \\
\hline$\geq 50$ & 858 & $259,651.83$ & 3.30 & 621 & $495,044.97$ & 1.25 & $2.37(2.12-2.64)^{c}$ & $1.34(1.20-1.50)^{c}$ \\
\hline \multicolumn{9}{|c|}{ Comorbidity } \\
\hline No & 19 & $20,949.87$ & 0.91 & 43 & $127,923.68$ & 0.34 & $2.41(1.40-4.14)^{\mathrm{e}}$ & $1.78(1.03-3.06)^{f}$ \\
\hline Yes & 1091 & $303,176.33$ & 3.59 & 708 & $476,627.31$ & 1.49 & $2.18(1.97-2.41)^{c}$ & $1.34(1.22-1.49)^{c}$ \\
\hline
\end{tabular}

PYs person-years, Rate incidence rate in per 1000 person-years, $95 \%$ Cl $95 \%$ confidence interval, HR hazard ratio

a Model adjusted for age, gender, Charlson Comorbidity Index and relevant comorbidities (hypertension, diabetes mellitus, hyperlipidemia, depression, asthma, allergic rhinitis, psoriasis, obesity, tobacco use disorder and alcohol attributed disease)

b Patients with any examined comorbidities, including hypertension, diabetes mellitus, hyperlipidemia, depression, asthma, allergic rhinitis, psoriasis, obesity, tobacco use disorder and alcohol attributed disease, were classified as the comorbidity group

c $\mathrm{P}<0.001$

d $P=0.003$

e $P=0.001$

$f^{P}=0.037$

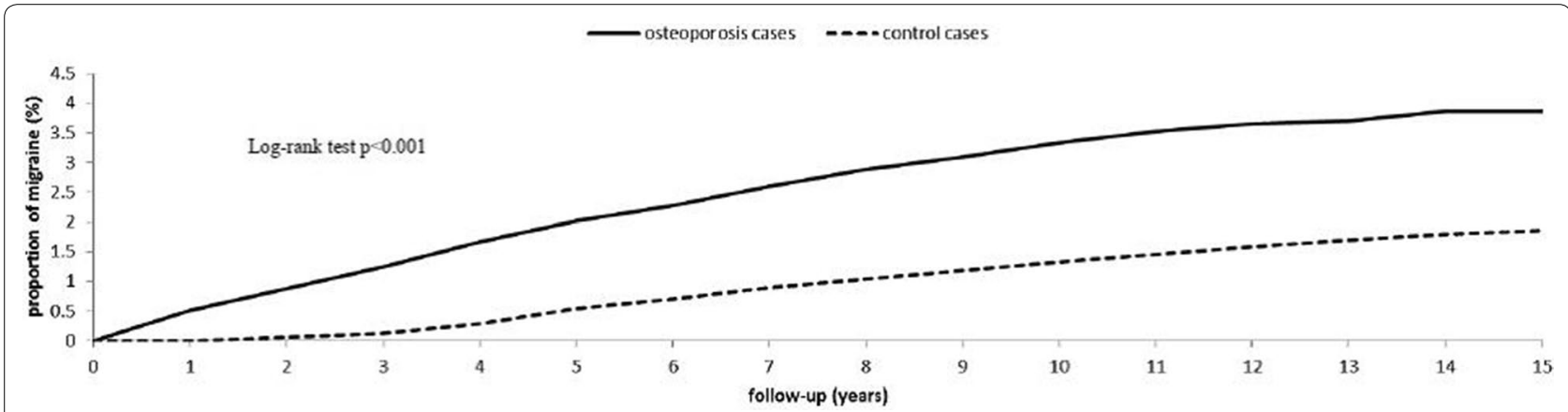

Fig. 2 Cumulative incidence of migraine for adult patients with osteoporosis and the general population control cohort

follow-up period, migraine developed in $2.73 \%$ (1110) patients with osteoporosis and in $1.85 \%$ (750) patients without osteoporosis. After controlling for potential confounding factors, migraine risk was 1.37 -fold higher in the osteoporosis group than in the non-osteoporosis group. Patients with osteoporosis, particularly those with high CCI score, female gender, hypertension, depression, asthma, allergic rhinitis, obesity, and tobacco use disorder, had a high migraine risk.

The exact mechanisms underlying the relationship between migraine and osteoporosis are likely to be elusive. However, several lines of evidence in the literature suggest that osteoporosis and migraine have a shared pathophysiology. First, bone density is significantly associated with magnesium, an essential micronutrient with a wide range of metabolic, structural and regulatory functions (Jahnen-Dechent and Ketteler 2012). In humans, magnesium deficiency contributes to osteoporosis. Low serum magnesium is a co-contributing factor in osteopenia in adults with sickle cell anemia (Elshal et al. 2012). Moreover, an association between serum magnesium and bone density has been identified in pre- and postmenopausal women (Saito et al. 2004; Song et al. 2007). Magnesium deficiency also has a strong association with migraine attacks (Welch and Ramadan 1995). Gallai et al. showed that individuals suffering from migraine 
Table 3 Cox regression model: significant predictors of migraine after osteoporosis

\begin{tabular}{lllr}
\hline Variables & Adjusted $^{\mathbf{H}} \mathbf{a}^{\mathbf{a}}$ & $\mathbf{( 9 5} \mathbf{\%} \mathbf{C l})$ & $\boldsymbol{P}$ value \\
\hline Age & 0.76 & $(0.72-0.81)$ & $<0.001$ \\
Charlson Comorbidity Index & 1.57 & $(1.44-1.71)$ & $<0.001$ \\
Female gender & 1.51 & $(1.23-1.85)$ & $<0.001$ \\
Hypertension & 1.19 & $(1.02-1.38)$ & 0.027 \\
Depression & 2.36 & $(2.09-2.66)$ & $<0.001$ \\
Asthma & 1.28 & $(1.13-1.45)$ & 0.001 \\
Allergic rhinitis & 1.46 & $(1.29-1.66)$ & $<0.001$ \\
Obesity & 1.79 & $(1.40-2.28)$ & $<0.001$ \\
Tobacco use disorder & 2.30 & $(1.78-2.97)$ & $<0.001$ \\
\hline
\end{tabular}

The adjusted HR and $95 \% \mathrm{Cl}$ were estimated by a stepwise the Cox proportional hazards regression method

$H R$ hazard ratio, $95 \%$ Cl $95 \%$ confidence interval

a Model adjusted for age, gender, Charlson Comorbidity Index and relevant comorbidities (hypertension, diabetes mellitus, hyperlipidemia, depression, asthma, allergic rhinitis, psoriasis, obesity, tobacco use disorder and alcohol attributed disease)

headaches had lower plasma and saliva magnesium levels between the attacks compared to controls without migraine headaches (Gallai et al. 1992). Both osteoporosis and migraine are associated with hypomagnesemia, which suggests an interplay between osteoporosis and migraine. Second, the relationship between migraine and osteoporosis might be explained at least partly by their common inflammatory mediators. Neurogenic inflammation resulting from activation of the trigeminal vascular system is the main cause of the pain produced by migraine. Stimulation of trigeminal ganglion nociceptors induces the release of proinflammatory substances, particularly calcitonin gene-related peptide (CGRP) (Silberstein 2004; Dalkara et al. 2006; D'Andrea and Leon 2010). Individuals with osteoporosis also had elevated CGRP levels (Lin et al. 2001). Experimental injections of CGRP into eviratated rats indicate that CGRP may also affect the release of osteoblastic cytokines and may indi-rectly regulate the suppressed bone resorption of osteoclasts (Valentijn et al. 1997). Inflammatory cytokines associated with osteoporosis such as tumor necrosis factoraand IL-6 (Braun and Schett 2012; Wiseman et al. 2014) are elevated at the onset of migraine attacks (Perini et al. 2005). Finally, C-reactive protein, which increases during systemic inflammation, is elevated in both osteoporosis and migraine (Vanmolkot and de Hoon 2007; de Pablo et al. 2012). Thus, the inflammatory state caused by osteoporosis may increase the frequency or severity of migraine headaches by exacerbating the inflammatory response.

The strength of our study is the use of a large sample that is highly representative of the general population and provides sufficient statistical power to identify an association between osteoporosis and migraine risk. However, several limitations must be considered when interpreting these findings. One limitation is that the analysis only included observational data, i.e., ICD9-CM codes, the accuracy of which depends on the clinical performance of individual physicians. That is, the accuracy of diagnostic codes in the database is a potential limitation. Notably, however, the Taiwan National Health Insurance program requires all insurance claims to be reviewed and audited by medical reimbursement specialists in the Bureau of National Health Insurance, which supports the validity and accuracy of the observed associations between osteoporosis and migraine. Furthermore, many studies have already used the NHIRD database because of its large size and long follow-up period (Chiu et al. 2015; Chu et al. 2015; Wu et al. 2015, 2016a, b, c, d). A second limitation is that the NHIRD does not contain detailed data that can be used to identify osteoporosis risk factors such as exercise capacity, body mass index, smoking, alcohol consumption, and dietary habits. A third limitation is that the Taiwan population analysed in this study was mostly of Chinese descent. Therefore, caution is needed when extrapolating the results to other ethnic groups; further studies are needed to determine whether these findings can be generalized to other ethnicities. Finally, this retrospective cohort study may have been biased by unrecognized or unadjusted confounding variables, despite the use of statistical methods for reducing their confounding effects.

\section{Conclusions}

In summary, this nationwide population-based cohort study revealed that adult patients with osteoporosis had a significantly higher risk of developing subsequent migraine compared to controls without osteoporosis. Clinicians should be aware that osteoporosis is a potential risk factor for migraine. Further studies are recommended to confirm this association and to explore its mechanisms.

\section{Abbreviations}

BMD: bone mineral density; CGRP: calcitonin gene-related peptide; CCI: Charlson Comorbidity Index; Cl: confidence interval; HR: hazard ratio; ICD-9-CM: International Classification of Diseases, Ninth Revision, Clinical Modification; L: interleukin; NHIRD: National Health Insurance Research Database.

\section{Authors' contributions}

CHW participated in study design, interpreted result and drafted the manuscript. ZHZ participated in study design and performed the statistical analysis. MKW participated in study design and helped to draft the manuscript. CHW helped to perform the statistical analysis and coordination. YYL and CLL conceived of the study, participated in its design, coordination and revising the manuscript. All authors read and approved the final manuscript. 


\begin{abstract}
Author details
1 Department of Dermatology, Kaohsiung Veterans General Hospital, No. 386 Dazhong 1st Rd, Kaohsiung 81362, Taiwan. ${ }^{2}$ Cosmetic Applications and Management Department, Yuh-Ing Junior College of Health Care and Management, Kaohsiung, Taiwan. ${ }^{3}$ Graduate Institute of Medicine, College of Medicine, Kaohsiung Medical University, Kaohsiung, Taiwan. ${ }^{4}$ Department of Neurosurgery, Kaohsiung Medical University Hospital, Kaohsiung Medical University, No. 100 Tzyou 1st Road, Kaohsiung 80708, Taiwan. ${ }^{5}$ Department of Neurosurgery, The No. 7 People's Hospital of Hebei Province, Dingzhou 073000, Hebei, People's Republic of China. ${ }^{6}$ Department of Psychiatry, Kaohsiung Chang Gung Memorial Hospital and Chang Gung University College of Medicine, Kaohsiung 807, Taiwan. ${ }^{7}$ Department of Nursing, Kaohsiung Medical University Hospital, Kaohsiung Medical University, No. 100 Tzyou 1st Road, Kaohsiung 80708, Taiwan. ${ }^{8}$ Department of Neurosurgery, Faculty of Medicine, College of Medicine, Kaohsiung Medical University, Kaohsiung, Taiwan
\end{abstract}

\section{Competing interests}

All authors declare that they have no competing interests.

Received: 12 July 2016 Accepted: 17 August 2016

Published online: 22 August 2016

\section{References}

Baykan B, Ertas M, Karli N, Uluduz D, Uygunoglu U, Ekizoglu E, Kocasoy Orhan E, Saip S, Zarifoglu M, Siva A (2015) Migraine incidence in 5 years: a populationbased prospective longitudinal study in Turkey. J Headache Pain 16:103

Bigal ME, Kurth T, Santanello N, Buse D, Golden W, Robbins M, Lipton RB (2010) Migraine and cardiovascular disease: a population-based study. Neurology 74(8):628-635

Braun T, Schett G (2012) Pathways for bone loss in inflammatory disease. Curr Osteoporos Rep 10(2):101-108

Charles A (2013) The evolution of a migraine attack - a review of recent evidence. Headache 53(2):413-419

Chiu YC, Hu HY, Lee FP, Huang HM (2015) Tension-type headache associated with obstructive sleep apnea: a nationwide population-based study. J Headache Pain 16:34

Cho SJ, Chung YK, Kim JM, Chu MK (2015) Migraine and restless legs syndrome are associated in adults under age fifty but not in adults over fifty: a population-based study. J Headache Pain 16:554

Chu CH, Liu CJ, Lin LY, Chen TJ, Wang SJ (2015) Migraine is associated with an increased risk for benign paroxysmal positional vertigo: a nationwide population-based study. J Headache Pain 16:62

D'Andrea G, Leon A (2010) Pathogenesis of migraine: from neurotransmitters to neuromodulators and beyond. Neurol Sci 31 (Suppl 1):S1-S7

Dalkara T, Zervas NT, Moskowitz MA (2006) From spreading depression to the trigeminovascular system. Neurol Sci 27(Suppl 2):S86-S90

de Pablo P, Cooper MS, Buckley CD (2012) Association between bone mineral density and C-reactive protein in a large population-based sample. Arthritis Rheum 64(8):2624-2631

Egeberg A, Mallbris L, Hilmar Gislason G, Skov L, Riis Hansen P (2015) Increased risk of migraine in patients with psoriasis: a Danish nationwide cohort study. J Am Acad Dermatol 73(5):829-835

Elshal MF, Bernawi AE, Al-Ghamdy MA, Jalal JA (2012) The association of bone mineral density and parathyroid hormone with serum magnesium in adult patients with sickle-cell anaemia. Arch Med Sci 8(2):270-276

Gallai V, Sarchielli P, Coata G, Firenze C, Morucci P, Abbritti G (1992) Serum and salivary magnesium levels in migraine. Results in a group of juvenile patients. Headache 32(3):132-135

Goadsby PJ, Lipton RB, Ferrari MD (2002) Migraine-current understanding and treatment. N Engl J Med 346(4):257-270

Headache Classification Subcommittee of the International Headache (2004) The International Classification of Headache Disorders: 2nd edition. Cephalalgia 24(Suppl 1):9-160

Hyder JA, Allison MA, Barrett-Connor E, Detrano R, Wong ND, Sirlin C, Gapstur SM, Ouyang P, Carr JJ, Criqui MH (2010) Bone mineral density and atherosclerosis: the Multi-Ethnic Study of Atherosclerosis, Abdominal Aortic Calcium Study. Atherosclerosis 209(1):283-289
Jahnen-Dechent W, Ketteler M (2012) Magnesium basics. Clin Kidney J 5(Suppl 1):i3-i14

Kalaydjian A, Merikangas K (2008) Physical and mental comorbidity of headache in a nationally representative sample of US adults. Psychosom Med 70(7):773-780

Kelman L, Rains JC (2005) Headache and sleep: examination of sleep patterns and complaints in a large clinical sample of migraineurs. Headache 45(7):904-910

Kim J, Cho SJ, Kim WJ, Yang KI, Yun CH, Chu MK (2016) Excessive daytime sleepiness is associated with an exacerbation of migraine: a populationbased study. J Headache Pain 17(1):62

Kuo WY, Huang CC, Weng SF, Lin HJ, Su SB, Wang JJ, Guo HR, Hsu CC (2015) Higher migraine risk in healthcare professionals than in general population: a nationwide population-based cohort study in Taiwan. J Headache Pain 16:102

Lampl C, Thomas H, Stovner LJ, Tassorelli C, Katsarava Z, Lainez JM, LanteriMinet M, Rastenyte D, Ruiz de la Torre E, Andree C, Steiner TJ (2016) Interictal burden attributable to episodic headache: findings from the Eurolight project. J Headache Pain 17:9

Lin J, Lu C, Gao L (2001) Study on the level of plasma calcitonin gene-related peptide and adrenomedullin in subjects with primary osteoporosis. Zhonghua Yi Xue Za Zhi 81(14):841-843

Manandhar K, Risal A, Linde M, Steiner TJ (2015a) The burden of headache disorders in Nepal: estimates from a population-based survey. J Headache Pain 17(1):3

Manandhar K, Risal A, Steiner TJ, Holen A, Linde M (2015b) The prevalence of primary headache disorders in Nepal: a nationwide population-based study. J Headache Pain 16:95

Mbewe E, Zairemthiama P, Paul R, Birbeck GL, Steiner TJ (2015) The burden of primary headache disorders in Zambia: national estimates from a population-based door-to-door survey. J Headache Pain 16:513

$\mathrm{NIH}$ Consensus Development Panel on Osteoporosis Prevention and Therapy (2001) Osteoporosis prevention, diagnosis, and therapy. JAMA 285(6):785-795

Perini F, D'Andrea G, Galloni E, Pignatelli F, Billo G, Alba S, Bussone G, Toso V (2005) Plasma cytokine levels in migraineurs and controls. Headache 45(7):926-931

Pompili M, Di Cosimo D, Innamorati M, Lester D, Tatarelli R, Martelletti P (2009) Psychiatric comorbidity in patients with chronic daily headache and migraine: a selective overview including personality traits and suicide risk. J Headache Pain 10(4):283-290

Rachner TD, Khosla S, Hofbauer LC (2011) Osteoporosis: now and the future. Lancet 377(9773):1276-1287

Radaei F, Darvishi A, Gharibzadeh S (2014) The correlation between osteoporosis occurrences in both schizophrenia and Parkinson's disease. Front Neurol 5:83

Rao GN, Kulkarni GB, Gururaj G, Stovner LJ, Steiner TJ (2015) The burden attributable to headache disorders in India: estimates from a communitybased study in Karnataka State. J Headache Pain 16:94

Risal A, Manandhar K, Holen A, Steiner TJ, Linde M (2016) Comorbidities of psychiatric and headache disorders in Nepal: implications from a nationwide population-based study. J Headache Pain 17:45

Sacco S, Ripa P, Grassi D, Pistoia F, Ornello R, Carolei A, Kurth T (2013) Peripheral vascular dysfunction in migraine: a review. J Headache Pain 14:80

Saito N, Tabata N, Saito S, Andou Y, Onaga Y, Iwamitsu A, Sakamoto M, Hori T, Sayama H, Kawakita T (2004) Bone mineral density, serum albumin and serum magnesium. J Am Coll Nutr 23(6):701S-703S

Schurks M, Rist PM, Bigal ME, Buring JE, Lipton RB, Kurth T (2009) Migraine and cardiovascular disease: systematic review and meta-analysis. BMJ 339:b3914

Silberstein SD (2004) Migraine pathophysiology and its clinical implications. Cephalalgia 24(Suppl 2):2-7

Song Y, Li TY, van Dam RM, Manson JE, Hu FB (2007) Magnesium intake and plasma concentrations of markers of systemic inflammation and endothelial dysfunction in women. Am J Clin Nutr 85(4):1068-1074

Steiner TJ, Birbeck GL, Jensen RH, Katsarava Z, Stovner LJ, Martelletti P (2015) Headache disorders are third cause of disability worldwide. J Headache Pain 16:58

Steyers CM 3rd, Miller FJ Jr (2014) Endothelial dysfunction in chronic inflammatory diseases. Int J Mol Sci 15(7):11324-11349 
Sumino H, Ichikawa S, Kasama S, Takahashi T, Sakamoto H, Kumakura H, Takayama Y, Kanda T, Murakami M, Kurabayashi M (2008) Relationship between carotid atherosclerosis and lumbar spine bone mineral density in postmenopausal women. Hypertens Res 31(6):1191-1197

Valentijn K, Gutow AP, Troiano N, Gundberg C, Gilligan JP, Vignery A (1997) Effects of calcitonin gene-related peptide on bone turnover in ovariectomized rats. Bone 21(3):269-274

Vanmolkot FH, de Hoon JN (2007) Increased C-reactive protein in young adult patients with migraine. Cephalalgia 27(7):843-846

Vanmolkot FH, Van Bortel LM, de Hoon JN (2007) Altered arterial function in migraine of recent onset. Neurology 68(19):1563-1570

Welch KM, Ramadan NM (1995) Mitochondria, magnesium and migraine. J Neurol Sci 134(1-2):9-14

Wiseman S, Marlborough F, Doubal F, Webb DJ, Wardlaw J (2014) Blood markers of coagulation, fibrinolysis, endothelial dysfunction and inflammation in lacunar stroke versus non-lacunar stroke and non-stroke: systematic review and meta-analysis. Cerebrovasc Dis 37(1):64-75
Wu TH, Hu LY, Lu T, Chen PM, Chen HJ, Shen CC, Wen CH (2015) Risk of psychiatric disorders following trigeminal neuralgia: a nationwide populationbased retrospective cohort study. J Headache Pain 16:64

Wu CH, Chai CY, Tung YC, Lu YY, Su YF, Tsai TH, Tzou RD, Lin CL (2016a) Herpes zoster as a risk factor for osteoporosis: a 15-year nationwide populationbased study. Medicine (Baltimore) 95(25):e3943

Wu CH, Lu YY, Chai CY, Su YF, Tsai TH, Tsai FJ, Lin CL (2016b) Increased risk of osteoporosis in patients with erectile dysfunction: a nationwide population-based cohort study. Medicine (Baltimore) 95(26):e4024

Wu CH, Tung YC, Chai CY, Lu YY, Su YF, Tsai TH, Kuo KL, Lin CL (2016c) Increased risk of osteoporosis in patients with peptic ulcer disease: a nationwide population-based study. Medicine (Baltimore) 95(16):e3309

Wu CH, Tung YC, Lin TK, Chai CY, Su YF, Tsai TH, Tsai CY, Lu YY, Lin CL (2016d) Hip fracture in people with erectile dysfunction: a nationwide populationbased cohort study. PLoS ONE 11(4):e0153467

\section{Submit your manuscript to a SpringerOpen ${ }^{\odot}$ journal and benefit from:}

- Convenient online submission

- Rigorous peer review

- Immediate publication on acceptance

- Open access: articles freely available online

- High visibility within the field

- Retaining the copyright to your article 